\title{
Three-Year Follow-Up of a Single-Center Series of Patients with Device-Aided Left Atrial Appendage Closure and Strict Echocar- diographic Control
}

\section{José Ramón López-Mínguez ${ }^{1}$, Juan Manuel Nogales-Asensio ${ }^{1}$, Victoria Millán-Núñez ${ }^{1}$, Patricia Márquez-Márquez ${ }^{1}$, Bruno Blenga-Limpo ${ }^{1}$, Carmen García Corrales ${ }^{1}$, Diego Félix Arroyo-Moñino ${ }^{1}$, Concepción López-Fernández ${ }^{2}$, Eugenia Fuentes-Cañamero ${ }^{1}$, Reyes González-Fernández and Anto- nio Merchán-Herrera ${ }^{1}$}

${ }^{1}$ Sección de Hemodinámica y Cardiología Intervencionista, Hospital Infanta Cristina, Badajoz, Spain

${ }^{2}$ Medicine Faculty, UAX, Madrid, Spain

*Corresponding author: José Ramón López-Mínguez, Sección de Hemodinámica y Cardiología Intervencionista, Hospital Infanta Cristina, Avda. Elvas s/n; Badajoz 06080, Spain, Tel: +349-242-181-25, E-mail: lopez-minguez@hotmail.com

\begin{abstract}
Introduction and objectives: Left atrial appendage closure (LAAC) is indicated in patients with non-valvular atrial fibrillation (NVAF) and contraindication for chronic anticoagulant therapy (OAC).

Methods: We evaluated the long-term progress of 78 consecutive patients after successful device-aided LAAC with transesophageal echocardiography (TEE) at $24 \mathrm{~h}, 1,3,6$, 12, 24 and 32 months.

Results: Mean patient age was $74.3 \pm 9.4$ years; $48.2 \%$ were women. Thirty patients $(37.4 \%)$ had paroxysmal AF and $53(62.7 \%)$ had persistent AF. LAAC was indicated in most patients $(73.5 \%)$ due to a contraindication for chronic anticoagulant treatment along with a history of bleeding. Median baseline embolic risk was 3 according CHADS scale and 5 according to the $\mathrm{CHA}_{2} \mathrm{DS}_{2}$-VASc score. Median bleeding risk according to the HAS-BLED score was 4. Mean follow-up was 32.2 months (209 patient-years). Event rate in 100 patients/year was death $9.1 \%$, severe bleeding $6.7 \%$ (7.7\% predicted by HAS-BLED), and stroke $2.4 \%\left(10 \%\right.$ or $9 \%$ predicted by $\mathrm{CHADS}_{2}, 5.9 \%$ or $6.7 \%$ by $\mathrm{CHA}_{2} \mathrm{DS}_{2}-\mathrm{VASc}$, Friberg and Lip respectively). Previous gastrointestinal bleeding revealed a HR of $4(p=0.04)$ for new bleed after surgery.
\end{abstract}

TEE revealed device-related thrombus or leak in 27 patients $(34.6 \%)$, associated with a higher incidence of stroke $(14.8 \%$ vs. $2.0 \% ; p=0.046)$.
Conclusions: LAAC is safe and effective in patients with NVAF and OAC contraindication. At 32 months, the thrombotic events rate reduced by $73 \%$ to $76 \%$ from $\mathrm{CHADS}_{2}$ or $\mathrm{CHA}_{2} \mathrm{DS}_{2}-\mathrm{VASC}$ predictions. Device-related thrombus or leaks may be associated with an increased incidence of stroke. Gastrointestinal bleeds are highly predictive of new bleeds during the first year.

\section{Keywords}

Left atrial appendage closure, Oral anticoagulants, Bleeding risk, Thromboembolic risk

\begin{abstract}
Abbreviations
AAl: Apéndice Auricular Izquierdo; FANV: Fibrilación Auricular No Valvular; OAC: Anticoagulante Oral; HGI: Hemorragia Gastrointestinal; AAS: Ácido Acetilsalicílico; RIC: Rango Intecuartílico; AAl: Left Atrial Appendage; NVAF: Non-Valvular Atrial Fibrillation; OAC: Oral Anticoagulant; GIB: Gastrointestinal Bleeding; ASA: Acetylsalicylic Acid; IQR: Interquartile Range
\end{abstract}

\section{Introduction}

Non-valvular atrial fibrillation (NVAF) is a significant health problem, particularly in the older population [1]. Since the introduction of novel oral anticoagulants (novel $\mathrm{OAC}$ ), greater numbers of patients at risk of stroke who previously were not candidates for oral anticoagulation $(O A C)$ with warfarin or acenocumarol due to the

Citation: López-Mínguez JR, Nogales-Asensio JM, Millán-Núñez V, Márquez-Márquez P, Blenga-Limpo B, et al. (2018) Three-Year Follow-Up of a Single-Center Series of Patients with Device-Aided Left Atrial Appendage Closure and Strict Echocardiographic Control. Int J Clin Cardiol 5:114. doi.org/10.23937/23782951/1410114

Accepted: May 30, 2018; Published: June 01, 2018

Copyright: (C) 2018 López-Mínguez JR, et al. This is an open-access article distributed under the terms of the Creative Commons Attribution License, which permits unrestricted use, distribution, and reproduction in any medium, provided the original author and source are credited. 
risk of bleeding are now being treated. Until then, up to $30 \%-40 \%$ of patients who needed OAC treatment were not treated due to fear of bleeding on the part of the patient or the physician, yet this group includes patients with higher risk of stroke, who often have a greater risk of bleeding complications [2]. Despite advances in the area of novel OAC in recent years, the number of atrisk patients who have experienced bleeding with OACS or novel OACs or who refuse to continue anticoagulant treatment remains high $[3,4]$. Left atrial appendage (LAA) closure has been accepted as a therapeutic strategy in these patients. Results from long-term follow-up after closure in patients who could receive OACs have demonstrated reduced mortality and events. Moreover, in patients who were not candidates for OACs, several registries have found fewer thromboembolic and bleeding events than predicted in follow-ups of longer than 1 year, with terms of $\mathrm{CHADS}_{2}, \mathrm{CHA}_{2} \mathrm{DS}_{2}$-VASC and HASBLED scores [5].

We report the results of a 32-month follow-up conducted in a single-center series of patients after LAA closure. Our patients underwent very close clinical and echocardiographic follow-up procedures, and special attention was paid to determining events reduction and predictive factors for bleeding and stroke.

\section{Materials and Methods}

\section{Patients and procedures}

This article presents the retrospective follow-up data from 78 NVAF patients of the 81 with successful LAA closure performed in our hospital between March 2, 2009 and December 16, 2015, that presented no complications and in whom OACs were contraindicated (implantation was unsuccessful in one and 2 presented local vascular complications that were successfully resolved). All patients signed informed consent for the intervention and for the follow-up, and the study protocol was approved by the ethics committee of our hospital.

Transesophageal echocardiography (TEE) was performed in all patients 24-48 hours before the intervention to rule out any thrombus within the LAA. The same operator performed all procedures and no incidents occurred during transeptal puncture.

A loading dose of clopidogrel $(600 \mathrm{mg})$ was administered for anticoagulation after implantation. Aspirin began with an initial $300 \mathrm{mg}$ dose on the first day after the intervention, followed by $100 \mathrm{mg}$ daily. Clopidogrel was maintained for 3 months, except in the case of bleeding complications, and aspirin was administered for at least 6 months.

Close clinical follow-up was performed with TEE at $24 \mathrm{~h}$, and 1, 3, 6 and 12 months. In case of thrombus, subcutaneous enoxaparin at therapeutic doses was added for 2 weeks, and the TEE was repeated to confirm that the thrombus had resolved. If it persisted, either treatment was extended by 1 week or the patient was admitted for administration of intravenous heparin, depending on clinical judgement.

\section{Variables and definitions}

Patients' baseline embolic risk was calculated according to the $\mathrm{CHADS}_{2}$ and $\mathrm{CHA}_{2} \mathrm{DS}_{2}$-VASc scores, with separate analysis based on the series by Lip [6] and Friberg [7]. Bleeding risk profile was calculated using the HAS-BLED score. In each follow-up visit, clinical events, particularly all-cause death and cardiac death, were evaluated and thrombotic and bleeding events requiring admission, with or without transfusion, were evaluated. Presence of device-associated thrombus, peri-device leak, residual interatrial communication (IAC), and correct positioning of the device were recorded during the follow-up TEE.

Thrombus was defined as echocardiographic density visible in more than 1 plane, which was either pedunculated and/or did not correspond to normal laminar reendothelialization of the exterior coating of the device. Identification was confirmed by consensus of 2 expert echocardiographers. Leak was interpreted as persisting flow $>1 \mathrm{~mm}$ around the edge of the device, passing into the appendage.

Bleeds were defined as severe/significant if they required admission or transfusion, if they caused a reduction of hemoglobin $>3 \mathrm{~g} / \mathrm{dl}$, or if bleeding was intracranial.

Observed incidence of events was calculated per patient and year of follow-up (number of patients at the beginning of the follow-up period multiplied by the mean time of follow-up of those patients expressed in years). The expected incidence of events in the sample was calculated as the mean of the individual risk of each patient.

\section{Statistical analysis}

Continuous variables were expressed as median (interquartile range, IQR). Categorical variables were compared using the $\chi^{2}$ or Fisher's exact test and numerical variables were compared using the Student $t$ or Wilcoxon tests. Comparisons between observed and expected rates of thromboembolic events and bleeds were evaluated using binomial tests. Event-free survival analysis was performed using Kaplan-Meier and Cox regression methods. All analyses were performed using the SPSS statistical package, version 19.0.

\section{Results}

\section{Implantation}

Of the 78 patients included, 48 received the Amplatzer $^{\mathrm{TM}}$ Cardiac Plug (ACP) and the next 30 received the Amplatzer ${ }^{\mathrm{TM}}$ Amulet (both from St Jude Medical, Minnesota, USA). Device size was selected according the manufacturer's tables, depending on the length of the implantation area, determined by consensus according 
to TEE measurement, angiography, and CT angiography at $10 \mathrm{~mm}$ and $12 \mathrm{~mm}$ from the ostium for the ACP and the Amulet, respectively.

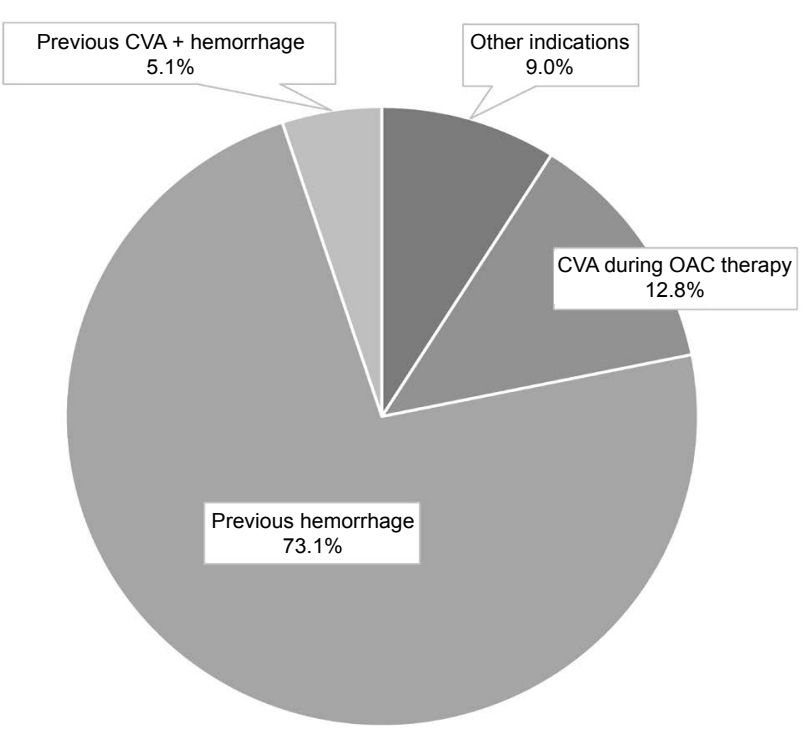

Figure 1: Indication for left atrial appendage closure.

\section{Baseline characteristics, risk profiles, and indications for closure}

Mean patient age was 77.8 years (interquartile range [IQR]: $67.9-81.0$, range: $46.0-89.9$ ). A total of $48.2 \%$ were women. The women were significantly older than the men (Figure 1). Baseline patient characteristics are shown in Table 1. Baseline patient characteristics, relevant history and embolic risk profiles ( $\mathrm{CHADS}_{2}$ and $\mathrm{CHA}_{2} \mathrm{DS}_{2}-\mathrm{VASC}$ ) and the bleeding risk profile (HAS-BLED) are shown in Table 1. The procedure was indicated in most patients due to a contraindication for chronic anticoagulant treatment because of a history of bleeding $(80.7 \%)$ or high bleeding risk (8.4\%). Previous most frequent bleed was gastrointestinal hemorrhage $(\mathrm{GIH})$ (43.4\%) (Table 2 and Figure 1).

\section{Clinical follow-up}

Median follow-up in the 78 patients with successful implantation was 24 months (IQR: 9-55). Table 3 shows clinical events during follow-up and Figure 2 the eventfree survival analysis until occurrence of stroke or se-

Table 1: Baseline demographic and clinical characteristics and baseline thromboembolic and hemorrhagic risk profiles.

\begin{tabular}{|c|c|c|c|c|}
\hline & All $(\mathrm{N}=78)$ & Men $(\mathrm{N}=43)$ & Women $(\mathrm{N}=40)$ & p-value \\
\hline Age in years (median and IQR) & $77.8[68.2-81.3]$ & $75.2[66.0-79.6]$ & $78.4[74.6-81.9]$ & 0.022 \\
\hline Paroxysmal AF & $25(32.1 \%)$ & $13(31.7 \%)$ & $12(32.4 \%)$ & 0.945 \\
\hline Permanent AF & $52(66.7 \%)$ & $28(68.3 \%)$ & $24(64.9 \%)$ & 0.748 \\
\hline \multicolumn{5}{|l|}{ Comorbidities } \\
\hline Arterial hypertension & $70(89.7 \%)$ & $37(90.2 \%)$ & $33(89.2 \%)$ & 0.878 \\
\hline Diabetes mellitus & $40(51.3 \%)$ & $17(41.5 \%)$ & $23(62.2 \%)$ & 0.068 \\
\hline \multicolumn{5}{|l|}{ Patient history } \\
\hline Previous PCI & $6(7.7 \%)$ & $3(7.3 \%)$ & $3(8.1 \%)$ & 0.954 \\
\hline Previous stroke & $18(23.1 \%)$ & $8(19.5 \%)$ & $10(27.0 \%)$ & 0.432 \\
\hline Previous stroke during OAC & $10(12.8 \%)$ & $4(9.8 \%)$ & $6(16.2 \%)$ & 0.341 \\
\hline Previous IHD & $18(23.1 \%)$ & $10(24.4 \%)$ & $8(21.6 \%)$ & 0.772 \\
\hline Previous CHF & $25(32.1 \%)$ & $14(34.1 \%)$ & $11(29.7 \%)$ & 0.676 \\
\hline \multicolumn{5}{|c|}{ Baseline embolic and bleeding risk profile } \\
\hline $\mathrm{CHADS}_{2}$ (median [IQR]) & $3[2-4]$ & $3[2-4]$ & $3[2-4]$ & 0.590 \\
\hline $\mathrm{CHA}_{2} \mathrm{DS}_{2}$-VASc (median [IQR]) & $5[3-5]$ & $4[3-5]$ & $5[4-6]$ & 0.005 \\
\hline HAS-BLED (median [IQR]) & $4[3-5]$ & $4[3-5]$ & $4[3-4]$ & 0.465 \\
\hline
\end{tabular}

Values are expressed as Number (percentage) unless otherwise indicated.

CHF: Congestive heart failure; CVA: Cerebrovascular accident; IHD: Ischemic heart disease; IQR: Interquartile range; OAC: Oral anticoagulant; $\mathrm{PCl}$ : Percutaneous cardiac intervention.

Table 2: Indication for procedure.

\begin{tabular}{|c|c|c|c|c|}
\hline & All ( $N=78)$ & $\operatorname{Men}(\mathrm{N}=43)$ & Women $(N=40)$ & $p$-value \\
\hline Stroke during $\mathrm{OAC}$ treatment & $10(12.8 \%)$ & $4(9.8 \%)$ & $6(16.2 \%)$ & 0.935 \\
\hline CVA + previous bleeding & $4(5.1 \%)$ & $2(4.9 \%)$ & $2(5.4 \%)$ & 0.930 \\
\hline Previous bleeding & $57(73.1 \%)$ & $28(68.3 \%)$ & $29(78.4 \%)$ & 0.821 \\
\hline Need for admission & $28(35.9 \%)$ & $15(36.6 \%)$ & $13(35.1 \%)$ & 0.894 \\
\hline Need for transfusion & $21(26.9 \%)$ & $13(31.7 \%)$ & $8(21.6 \%)$ & 0.316 \\
\hline \multicolumn{5}{|l|}{ Type of previous bleeding } \\
\hline Gastrointestinal bleeding & $33(42.3 \%)$ & $16(39.0 \%)$ & $17(45.9 \%)$ & 0.537 \\
\hline Intracranial hemorrhage & $15(19.2 \%)$ & $8(19.5 \%)$ & $7(18.9 \%)$ & 0.947 \\
\hline Other bleeding & $9(11.5 \%)$ & $4(9.8 \%)$ & $5(13.5 \%)$ & 0.748 \\
\hline Other indications ${ }^{*}$ & $7(9.0 \%)$ & $7(17.1 \%)$ & 0 & 1.000 \\
\hline
\end{tabular}

Values are expressed as number (percentage).

CVA: Cerebrovascular accident; $\mathrm{Hb}$ : Hemoglobin; OAC: Oral anticoagulant. "High risk of bleeding, poor INR control or patient's decision. 

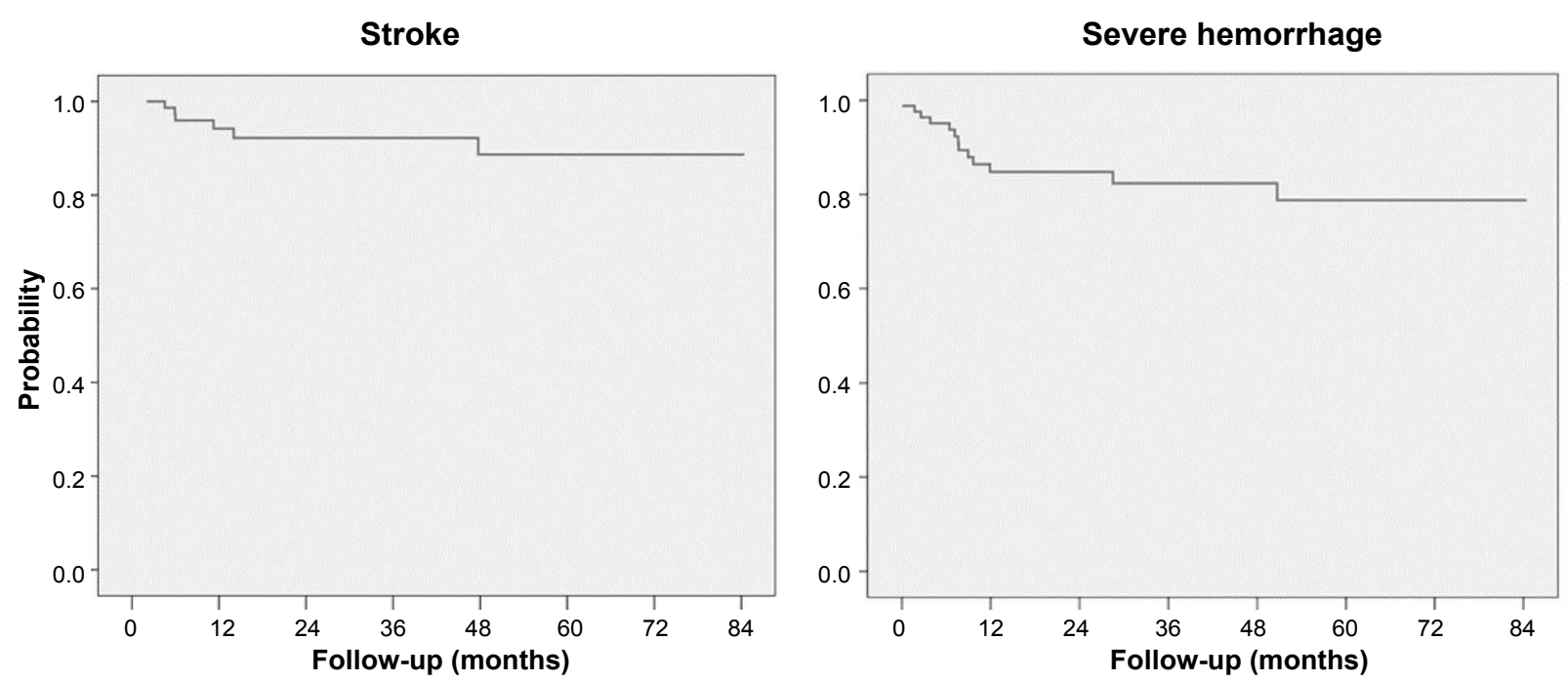

Figure 2: Post-procedure event-free survival Kaplan-Meier curves for "stroke" and "severe bleeding".

Table 3: Clinical events during clinical follow-up in patients with successful implantation.

\begin{tabular}{|c|c|c|c|c|}
\hline Events & All $(N=78)$ & Men $(\mathrm{N}=41)$ & Women $(\mathrm{N}=37)$ & p-value \\
\hline Death & $19(24.4 \%)$ & $8(19.5 \%)$ & $11(29.7 \%)$ & 0.225 \\
\hline Stroke & $5(6.4 \%)$ & $4(9.8 \%)$ & $1(2.7 \%)$ & 0.362 \\
\hline Systemic embolism & 0 & 0 & 0 & \\
\hline Bleeding & $16(20.5 \%)$ & $9(22.0 \%)$ & $7(18.9 \%)$ & 0.692 \\
\hline Gastrointestinal bleeding & $12(15.4 \%)$ & $7(17.1 \%)$ & $5(13.5 \%)$ & 0.625 \\
\hline Intracranial hemorrhage & 0 & 0 & 0 & \\
\hline Other bleeding & $4(5.1 \%)$ & $2(4.9 \%)$ & $2(5.4 \%)$ & 0.925 \\
\hline Severe/significant bleeding* & $14(17.9 \%)$ & $7(17.1 \%)$ & $7(18.9 \%)$ & 0.882 \\
\hline
\end{tabular}

"Defined as "need for admission", "need for transfusion" or "intracranial hemorrhage" or reduction in $\mathrm{Hb}>3 \mathrm{~g} / \mathrm{dl}$.

Table 4: Types of bleeding and time to appearance by antiplatelet treatment.

\begin{tabular}{|l|l|l|l|}
\hline Patient & Months post-implantation & Type of bleeding & Treatment at time of bleeding event \\
\hline RMH & 4 & Gastrointestinal & ASA + clopidogrel \\
\hline MMM & 10 & Gastrointestinal & ASA \\
\hline MLF & 1 & Gastrointestinal & ASA + clopidogrel \\
\hline SBL & 7 & Gastrointestinal & ASA \\
\hline PCJ & 6 & Unknown & ASA + clopidogrel \\
\hline EPF & 3 & Gastrointestinal & ASA + clopidogrel \\
\hline EBF & 1 & Gastrointestinal & ASA + clopidogrel \\
\hline RPM & 2 & Gastrointestinal & ASA + clopidogrel \\
\hline MGM & 4 & Gastrointestinal & ASA + clopidogrel \\
\hline RPJ & 1 & Gastrointestinal & ASA + clopidogrel \\
\hline AZMG & 4 & Gastrointestinal & ASA + clopidogrel \\
\hline
\end{tabular}

ASA: acetylsalicylic acid.

Table 5: Comparison of observed events with predicted events $(\mathrm{N}=78)$.

\begin{tabular}{|l|l|l|l|l|}
\hline Events (per 100 patients/year) & Observed & Predicted & Reduction & p-value \\
\hline Death & $19(9.1 \%)$ & & & 0.389 \\
\hline Severe/significant bleeding & $14(6.7 \%)$ & $7.7 \%(\mathrm{HAS}-\mathrm{BLED})$ & $(-13 \%)$ & \\
\hline Stroke & $5(2.4 \%)$ & $\left.\begin{array}{l}10.0 \%(\mathrm{CHADS} \\
2\end{array}\right)$ & $5.9 \%$ & $<0.001$ \\
& & $(-73 \%)$ & $(-59 \%)$ & $<0.001$ \\
\hline
\end{tabular}

*Overall follow-up: 32.2 months (209 patients/year).

vere hemorrhage. Most events occurred within the first year: Bleeding occurred 11 patients (14.1\%) of the overall 16 with hemorrhage, and this was during the dual antiplatelet treatment period. Bleeding was gastrointestinal (GI) in 10 patients (91\%) (Table 4). A history of GIH was associated with a greater frequency of major 
bleeds 1 year after implantation (HR: 4 HR: 4.16; IC95\%: 1.1 a $15.7 ; p=0.03$ ). There was a significant reduction in the incidence of stroke compared to expected values (Table 5).

\section{Echocardiographic follow-up}

Device-related thrombus or leak was observed on follow-up echocardiography in 27 of the 78 monitored patients (34.6\%). Leaks were less than $3 \mathrm{~mm}$ in all cases. These patients had a greater incidence of stroke $(14.8 \%$ vs. $2.0 \% ; p=0.046$ ) compared to those who did not present leaks (Figure 3 and Table 6).

\section{Discussion}

The main findings of our long-term follow-up study of patients after LAA closure due to OAC contraindication can be summarized in 3 main points. Firstly, we found a large and significant reduction in the number of thromboembolic events compared to the expected rate according to $\mathrm{CHADS}_{2}$ and $\mathrm{CHA}_{2} \mathrm{DS}_{2}$-VASc scores. Secondly, rates of device-associated thrombus and leaks peak between 3 and 6 months, precisely the period that is not examined in most studies, and the appearance of these events can predict a risk of thromboembolism in these patients when a very close follow-up is performed with transesophageal echocardiography. Thirdly, GI bleeding as an indication for left atrial appendage closure is the best predictor of bleeding during follow-up.

\section{Long-term reduction of thromboembolic events}

The patient-year incidence of stroke in our patients at 3 years was $2.4 \%$ vs. the $9 \%$ or $6.7 \%$ predicted by the $\mathrm{CHA}_{2} \mathrm{DS}_{2}-\mathrm{VASC}$ in previous series, with reductions of $64 \%-76 \%[6,7]$. These results are consistent with both single-center series $[8,9]$ and multicenter series, such as those of the Iberian Registry or the European ACP multicenter study and the ASAP registry with the Watchman device [10-12], in patients with contraindication for OAC. The patient-year incidence of stroke in the Iberian Registry was $3.9 \%$ at 1 year, and $2.4 \%$ at 2 years vs. $8.3 \%$ according to the $\mathrm{CHA}_{2} \mathrm{DS}_{2}$-VASc. These reductions are maintained throughout follow-up periods of up to 3-4 years, with very similar figures reported in the various studies.

Compared with warfarin, the studies PREVAIL and PROTECT AF, with Watchman device implantation in patients with no contraindication for OAC, showed a reduction in the incidence of stroke and a similar incidence of bleeding events compared to the anti-vitamin K $[13,14]$.

For obvious reasons, no randomized studies have been conducted in patients with a contraindication for OAC. It is thought that at least $20 \%-30 \%$ of the NVAF population fall within this group. Moreover, the rate of drop-outs or treatment discontinuations in randomized studies with novel OACs ranges from $21.2 \%$ to $33.5 \%$ [3]. These data suggest that half of NVAF patients who

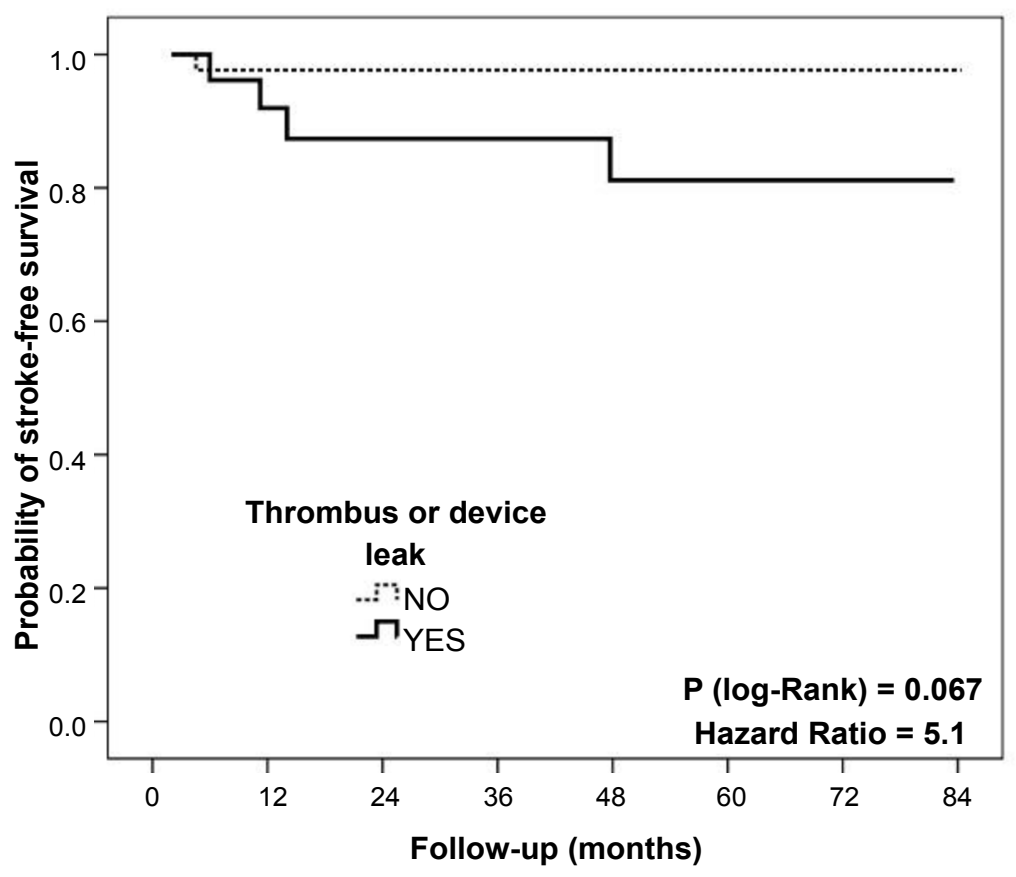

Figure 3: Event-free survival Kaplan-Meier curves for "stroke" during follow-up in patients with and without device-related thrombus on echocardiographic monitoring.

Table 6: Variables during echocardiographic follow-up in patients with successful implantation.

\begin{tabular}{|c|c|c|c|c|}
\hline & $24 h(n=75)$ & $1 m(n=60)$ & 3-6 m (n = 56) & $12 m(n=45)$ \\
\hline Thrombus, $\mathbf{n}(\%)$ & $3(4 \%)$ & $3(5 \%)$ & $5(8.9 \%)$ & $3(6.7 \%)$ \\
\hline Leak, n (\%) & $7(9.3 \%)$ & $8(13.3 \%)$ & $12(21.4 \%)$ & $6(13.3 \%)$ \\
\hline Residual Interatrial communication, $\mathbf{n}(\%)$ & $61(81.3 \%)$ & $37(61.7 \%)$ & $23(41.1 \%)$ & $10(22.2 \%)$ \\
\hline
\end{tabular}


require $\mathrm{OAC}$ will be receiving inappropriate treatment or their compliance will be insufficient to provide protection against stroke or thrombus [4]. In this setting, a certain proportion of patients with NVAF and contraindication for OAC continue to receive aspirin to prevent stroke, in the false belief that this molecule will provide protection with a lower risk of bleeding. Indeed, the results of the PINNACLE registry [15] showed that $39 \%$ of AF patients were taking aspirin only, despite an intermediate-high risk according to their $\mathrm{CHA}_{2} \mathrm{DS}_{2}-\mathrm{VASc}$ score. This strategy has already been revealed as erroneous by the BAFTA study [16]. During a mean follow-up of 2.7 years, 24 primary events (fatal or incapacitating ischemic or hemorrhagic stroke, intracranial hemorrhage, or systemic embolism) occurred in the warfarin group and 48 primary events occurred in the aspirin group (annual risk of $1.8 \%$ vs. $3.8 \%$, RR $0.48, p=0.003$; absolute reduction of annual risk: $2 \%$ ). Similar data were derived from the ACTIVE W study [17]. Thus, the data on LAA closure available to date do not justify this strategy.

\section{Prediction of stroke or thromboembolism in long- term follow-up}

Some studies addressing this issue have explored factors such as left ventricular dysfunction or platelet concentrations, but most studies have focused on the presence of device-associated thrombus and persistent leaks $[18,19]$.

The consensus among researchers for thrombi determination taken into account in our protocol is important. In PROTECT AF, of the initial 35 cases with thrombus (among 485 patients), 27 remained after strict consensus definition criteria [20]. The overall incidence of thrombus at any time during the period before the procedure and 1 year after was 5.7\%. Moreover, the percentages varied, being greater at 6 months (19 cases) and 12 months (12 cases, many of whom were different from those seen at 6 months), than at 45 days ( 7 cases). This supports the results of our study, indicating that a single time point is not at all reliable for determining the incidence of device-associated thrombus. Lower percentages of device-associated thrombus were reported in studies with fewer TEE follow-ups, while studies with stricter protocols found greater numbers of thrombus $[18,21]$. Of course, these percentages have fallen, thanks to greater experience.

Results of PROTECT AF reflect the fact that warfarin in the first 45 days may have a protective effect against the formation of thrombus not provided by aspirin and clopidogrel or aspirin alone after 6 months. The most interesting aspect of this analysis is that primary efficacy event rate (death, stroke, systemic embolism) in patients with thrombus was $3.4 \%$ patient-year, worse than the overall $2.2 \%$ for the device, but better than the $3.7 \%$ for patients on warfarin after 5 years of follow-up. The Iberian Registry reported a frequency of device-associated thrombus of $8.2 \%$, and these patients had a higher rate of stroke, although the difference was not significant (4.2 per 100 patients/year compared to 2.2 per 100 patients/year; HR: 1.9; $p=0.550$ ) [10].

In the PROTECT AF study, leaks were observed in $32.1 \%$ of patients at 12 months (at 6 months, the rate fell from $40.9 \%$ to $33.8 \%$ ). Patients took warfarin for the first 45 days, and only discontinued if no leak or leak $\leq 5 \mathrm{~mm}$ was seen on the TEE. TEEs were performed at 45 days, 6 months and 12 months. All patients received clopidogrel between day 45 and month 6 , and aspirin indefinitely. Curiously, patients with leaks had fewer thromboembolic events ( $2 \%$ vs. $2.8 \%$ ) but the difference was not significant. In this study, however, patients routinely received acenocoumarol for 45 days or for longer if leak was observed, compared to patients who did not present leak and therefore did not receive warfarin [22] It is interesting to note that only leaks $<1 \mathrm{~mm}$ tended to resolve after 1 year, which was not the case for leaks of 1-3 $\mathrm{mm}$ or $>3 \mathrm{~mm}$.

In the European multicenter series, 339 analyzable patients were included in the population with contraindication for OAC [11] with average cardiovascular and thromboembolic risks somewhat lower than those of our series, according to the three scores. Median clinical follow-up was 355 days (range 179-622), and follow-up TEE was performed after a median of 134 days (range 88-227). Device-associated thrombus was observed in $3.2 \%$ and peri-device leak occurred in $12.5 \%$. Neither device-associated thrombus or peri-device leak was associated with an increased risk of cardiovascular events. Leaks were observed in $7.8 \%$ of patients in the Iberian Registry [10].

In studies of populations with contraindication for OAC, warfarin was not administered, and fewer TEE procedures were performed during follow-up than in our study. The detection of echocardiographic events with strict monitoring in a single-center study may be more reliable than in multicenter studies operating with different protocols and study time points. In a single-center study, Bai, et al. studied a series of 58 consecutive patients implanted with a Watchman device. TEE was performed peri-procedurally and at 45 days and 12 months. Peri-device leak was found in $27.6 \%, 29.3 \%$ and $34.5 \%$ of cases, at each respective time point [23]. These variations are in line with our findings. In their series, 1 patient had device-associated thrombus, and another had ischemic stroke: Both of these patients had peri-device leak. These authors suggest that leaks may increase in size over time and persist, and that new leaks may even appear, most probably due to LAA remodeling. Cases with increased peri-device leak during follow-up coinciding with stroke during this period have also been reported [24].

Leaks appear to be less common with the Amplatzer devices than with the Watchman device. Urena, et al. reported a rate of $16.2 \%$ among 37 patients who under- 
went TEE in a series of 50 patients with Amplatzer in a 2-year follow-up: These figures are half those reported with the Watchman. On CT angiography, however, leak figures appear to be higher than on TEE. Saw, et al. described $62 \%$ of leaks, with no differences between devices and formed by different mechanisms [25]. Finally, incomplete LAA closure in patients undergoing cardiac surgery (10\%-70\%) has been revealed as a predictor of thromboembolic events in some series [26].

It should be pointed out that in most studies, the TEE follow-up is performed at 45 days and 6 months. The incidence of leaks or thrombus in our series at these time points is the same as in other studies with similar time points. It is interesting to note that the incidence of leaks and thrombus increases between 3 and 6 months, so published studies almost certainly underestimate the rate of these events, since these percentages can also vary over time. Keeping these considerations in mind, our study shows that patients who presented leak and/ or thrombus on close TEE monitoring had a higher risk of stroke. Our data must be regarded cautiously, due to the small number of cases and the fact that they do not coincide with the findings of most previously published studies. However, they do raise the possibility that patients with these signs should be monitored in shorter intervals and assess the need to use of a similar strategy to that of the PROTECT AF study should be evaluated in these cases, with the administration of warfarin or novel OACs maintained over several months [27].

\section{Prediction of bleeding events during follow-up}

In our series, 11 patients (14.1\%) had significant bleeding during the first year after implantation, mostly GIH and during dual antiplatelet treatment in the first 6-month Rates during follow-up were $6.7 \%$ patient-years; this figure was somewhat lower than the $7.7 \%$ predicted according to the HAS-BLED score. It is the usual scenario in these patients with contraindication to anticoagulants and high bleeding risk, as observed in the Iberian Registry, the European multicenter registry [10,11], and in EWOLUTION [28].

Bleeding rates in patients with contraindication for $\mathrm{OAC}$ in these registries is generally higher than those reported in clinical trials with patients without contraindications, but the rates of major bleeding in real life double those in trials, whereas $<70 \%$ of patients are eligible [29]. These data show that the risk of bleeding is high during the first year and particularly during the early months when dual antiplatelet treatment is being administered. Advantages in terms of reduced bleeding begin to be seen in the long term, after the 12-month point. This year need to be taken into consideration. In the Iberian Registry, after 1 year $88 \%$ of patients continued to receive at least 1 antiplatelet agent, generally aspirin. The BAFTA study already showed that bleeds in patients $>75$ years of age were practically the same with aspirin as with warfarin.
However, in the EWOLUTION [28] registry with Watchman device, post-intervention treatment with a novel OAC showed less bleeding events than other antiplatelet therapy strategies or warfarin, and even compared to patients without any treatment. In this study, history of GIH as a strong predictor of new GIH after implant. In these patients with OAC contraindication and previous $\mathrm{GIH}$, suspension of aspirin should be considered after the first 6 months, since GIH, especially in elderly population with $\mathrm{OAC}$, is a marker of high mortality. In a Danish study, 5-15\% of patients with OAC belonging to the general population had GIH [30]. After two years of discharge after $\mathrm{GIH}$, with an average age of 78 years, $40 \%$ of the patients had died, $17.7 \%$ experienced severe bleeding, and $12.1 \%$ a new GIH. However, it was better to restart the OAC treatment: Patients had more $\mathrm{GIH}$, but mortality was reduced. However, in other studies, up to $51 \%$ of patients do not restart OAC after a bleeding [31].

Most recurrent bleeding events occur in the first 90 days after $\mathrm{Gl}$ bleeding: In this period $8 \%$ presented $\mathrm{GI}$ bleeds, $4.7 \%$ thromboembolisms, and $9.2 \%$ of patients died. Similarly, Witt, et al. reported a rate of $10 \% \mathrm{Gl}$ bleeding in the first 90 days in patients who recommenced treatment. However, despite the presence of $5.5 \%$ recurrent $\mathrm{Gl}$ bleeding in those who did not start treatment, mortality was $5.8 \%$ vs. $20.3 \%$, and $0.4 \%$ vs. $5.5 \%$ had a thromboembolic event when OAC was reinitiated [32]. In patients undergoing percutaneous coronary interventions, most bleeding events in a study by Genereux, et al. were GI (61.7\%), and the greatest predictive factor was use of OAC (OR: 2.31). Bleeding impacted mortality 2.6 times more than acute myocardial infarction [33].

\section{Conclusions}

LAA closure is effective in reducing thromboembolic events in the first year and in the long term produces significant reductions of $>60 \%$ in events predicted by scores.

Echocardiographic monitoring between 3-6 months reveals a critical period in which more device-related thrombus or leaks can occur: Curiously, this period is omitted in the majority of the studies, explaining variations in the percentages of these events. The impact remains unclear, but it may be of more importance than previously thought.

GI bleeding is the best predictor of new bleeds, particularly in the first year, and especially in individuals older than 75 years of age who continue treatment with aspirin. After this critical year, however, reductions in bleeding events begin to be observed, compared to rates predicted by HAS-BLED score.

\section{Acknowledgements}

We thank Dr. Blanca Piedrafita of Medical Statistics 
Consulting for editing this manuscript. This collaborative effort was funded by St. Jude Medical.

\section{Key Points}

\section{What is known about the topic?}

The novel OAC have ensured that a greater number of patients at risk of stroke who previously took as traditional OAC, such as warfarin or acenocoumarol due to their risk of bleeding, is at present receiving treatment. However, the practice of using antiplatelet drugs instead of OAC persists in these patients, as the former are falsely believed to be safer.

Percutaneous closure of the left atrial appendage (AAI) is indicated in patients with non-valvular atrial fibrillation (NVAF) and contraindication for chronic anticoagulant therapy (OAC).

\section{What does it again?}

We present the monitoring results at 32 months of a single-center series of patients with AAI closure and contraindication for OAC, who had a very strict echocardiographic and clinical follow-up.

Percutaneous closure of AAl is a safe and effective technique in patients with NVAF not candidates for long-term anticoagulant therapy. However, the first 6 months is a critical period in which most events in patients treated with dual antiplatelet therapy occur.

History of gastrointestinal bleeding is highly predictive of new bleeding during the first year.

The presence of thrombi in the device or leak might require closer monitoring or restart short periods of anticoagulation.

\section{References}

1. Pérez-Villacastín J, Pérez Castellano N, Moreno Planas J (2013) Epidemiology of atrial fibrillation in Spain in the past 20 years. Rev Esp Cardiol (Engl Ed) 66: 561-565.

2. Pälomaki A, Mustonen P, Hartikainen JE, Nuotio I, Kiviniemi T, et al. (2016) Underuse of anticoagulation in stroke patients with atrial fibrillation--the FibStroke Study. Eur J Neurol 23: 133-139.

3. Ruff CT, Giugliano RP, Braunwald E, Hoffman EB, Deenadayalu N, et al. (2014) Comparison of the efficacy and safety of new oral anticoagulants with warfarin in patients with atrial fibrillation: A meta-analysis of randomised trials. Lancet 383: 955-962.

4. Yao X, Abraham NS, Alexander GC, Crown W, Montori VM, et al. (2016) Effect of Adherence to Oral Anticoagulants on Risk of Stroke and Major Bleeding Among Patients With Atrial Fibrillation. J Am Heart Assoc 5.

5. Li X, Wen SN, Li SN, Bai R, Liu N, et al. (2016) Over 1-year efficacy and safety of left atrial appendage occlusion versus novel oral anticoagulants for stroke prevention in atrial fibrillation: A systematic review and meta-analysis of randomized controlled trials and observational studies. Heart Rhythm 13: 1203-1214.

6. Lip GY, Frison L, Halperin JL, Lane DA (2011) Compar- ative validation of a novel risk score for predicting bleeding risk in anticoagulated patients with atrial fibrillation: the HAS-BLED (Hypertension, Abnormal Renal/Liver Function, Stroke, Bleeding History or Predisposition, Labile INR, Elderly, Drugs/Alcohol Concomitantly) score. J Am Coll Cardiol 57: 173-180.

7. Friberg L, Rosenqvist M, Lip GY (2012) Evaluation of risk stratification schemes for ischaemic stroke and bleeding in 182678 patients with atrial fibrillation: the Swedish Atrial Fibrillation cohort study. Eur Heart J 33: 1500-1510.

8. Urena M, Rodes-Cabau J, Freixa X, Saw J, Webb JG, et al. (2013) Percutaneous left atrial appendage closure with the AMPLATZER cardiac plug device in patients with nonvalvular atrial fibrillation and contraindications to anticoagulation therapy. J Am Coll Cardiol 62: 96-102.

9. Santoro G, Meucci F, Stolcova M, Rezzaghi M, Mori F, et al. (2016) Percutaneous left atrial appendage occlusion in patients with non-valvular atrial fibrillation: implantation and up to four years follow-up of the AMPLATZER Cardiac Plug. Eurolntervention 11: 1188-1194.

10. López Mínguez JR, Asensio JM, Gragera JE, Costa M, González IC, et al. (2015) Two-year clinical outcome from the Iberian registry patients after left atrial appendage closure. Heart 101: 877-883.

11. Tzikas A, Shakir S, Gafoor S, Omran H, Berti S, et al. (2016) Left atrial appendage occlusion for stroke prevention in atrial fibrillation: multicentre experience with the AMPLATZER Cardiac Plug. Eurolntervention 11: 1170-1179.

12. Reddy VY, Möbius-Winkler S, Miller MA, Neuzil P, Schuler G, et al. (2013) Left atrial appendage closure with the Watchman device in patients with a contraindication for oral anticoagulation: The ASAP study (ASA Plavix Feasibility Study With Watchman Left Atrial Appendage Closure Technology). J Am Coll Cardiol 61: 2551-2556.

13. Reddy VY, Sievert H, Halperin J, Doshi SK, Buchbinder M, et al. (2014) Percutaneous left atrial appendage closure vs warfarin for atrial fibrillation: a randomized clinical trial. JAMA 312: 1988-1998.

14. Holmes DR Jr, Doshi SK, Kar S, Price MJ, Sanchez JM, et al. (2015) Left Atrial Appendage Closure as an Alternative to Warfarin for Stroke Prevention in Atrial Fibrillation: A Patient-Level Meta-Analysis. J Am Coll Cardiol 65: 26142623.

15. Hsu JC, Maddox TM, Kennedy K, Katz DF, Marzec LN, et al. (2016) Aspirin Instead of Oral Anticoagulant Prescription in Atrial Fibrillation Patients at Risk for Stroke. J Am Coll Cardiol 67: 2913-2923.

16. Mant J, Hobbs FD, Fletcher K, Roalfe A, Fitzmaurice D, et al. (2007) Warfarin versus aspirin for stroke prevention in an elderly community population with atrial fibrillation (the Birmingham Atrial Fibrillation Treatment of the Aged Study, BAFTA): A randomised controlled trial. Lancet 370: 493503.

17. Connolly S, Pogue J, Hart R, Pfeffer M, Hohnloser S, et al. (2006) Clopidogrel plus aspirin versus oral anticoagulation for atrial fibrillation in the Atrial fibrillation Clopidogrel Trial with Irbesartan for prevention of Vascular Events (ACTIVE W): A randomised controlled trial. Lancet 367: 1903-1912.

18. Plicht B, Konorza TF, Kahlert P, Al-Rashid F, Kaelsch H, et al. (2013) Risk factors for thrombus formation on the Amplatzer Cardiac Plug after left atrial appendage occlusion. JACC Cardiovasc Interv 6: 606-613.

19. Saw J, Lempereur M (2014) Percutaneous left atrial ap- 
pendage closure: Procedural techniques and outcomes. JACC Cardiovasc Interv 7: 1205-1220.

20. Main ML, Fan D, Reddy VY, Holmes DR, Gordon NT, et al. (2016) Assessment of Device-Related Thrombus and Associated Clinical Outcomes with the WATCHMAN Left Atrial Appendage Closure Device for Embolic Protection in Patients with Atrial Fibrillation (from the PROTECT-AF Trial). Am J Cardiol 117: 1127-1134.

21. Park JW, Bethencourt A, Sievert H, Santoro G, Meier B, et al. (2011) Left atrial appendage closure with Amplatzer cardiac plug in atrial fibrillation: Initial European experience. Catheter Cardiovasc Interv 77: 700-706.

22. Viles-González JF, Kar S, Douglas P, Dukkipati S, Feldman T, et al. (2012) The clinical impact of incomplete left atrial appendage closure with the Watchman Device in patients with atrial fibrillation: A PROTECT AF (Percutaneous Closure of the Left Atrial Appendage Versus Warfarin Therapy for Prevention of Stroke in Patients With Atrial Fibrillation) substudy. J Am Coll Cardiol 59: 923-939.

23. Bai R, Horton RP, L DIB, Mohanty P, Pump A, et al. (2012) Intraprocedural and long-term incomplete occlusion of the left atrial appendage following placement of the WATCHMAN device: A single center experience. J Cardiovasc Electrophysiol 23: 455-461.

24. Lee K, Park SJ, Kwon HJ, Kim IY, Yoo KH, et al. (2014) Progressive increase in peridevice leakage after the implantation of the watchman device on long-term serial echocardiographic follow-up. Can J Cardiol 30: 1461.e151461.e17.

25. Saw J, Fahmy P, DeJong P, Lempereur M, Spencer R, et al. (2015) Cardiac CT angiography for device surveillance after endovascular left atrial appendage closure. Eur Heart J Cardiovasc Imaging 16: 1198-1206.

26. García-Fernández MA, Pérez-David E, Quiles J, Peralta J, García-Rojas I, et al. (2003) Role of left atrial appendage obliteration in stroke reduction in patients with mitral valve prosthesis: A transesophageal echocardiographic study. J Am Coll Cardiol 42: 1253-1258.

27. Holmes DR, Reddy VY, Turi ZG, Doshi SK, Sievert H, et al. (2009) Percutaneous closure of the left atrial appendage versus warfarin therapy for prevention of stroke in patients with atrial fibrillation: A randomised non-inferiority trial. Lancet 374: 534-542.

28. Bergmann MW, Schmidt B, Betts T (2016) EWOLUTION: 3-months outcome of left atrial appendage closure with the Watchman device in Europe: thrombus at the device, leakage at follow-up, device embolisation, post-procedural drug regimen and impact of center experience data from the prospective 1025 patients EWOLUTION Registry. EuroPCR Paris, France.

29. Levi M, Hovingh K (2008) Bleeding complications in patients on anticoagulants who would have been disqualified for clinical trials. Thromb Haemost 100: 1047-1051.

30. Staerk L, Lip GY, Olesen JB, Fosbol EL, Pallisgaard JL, et al. (2015) Stroke and recurrent haemorrhage associated with antithrombotic treatment after gastrointestinal bleeding in patients with atrial fibrillation: Nationwide cohort study. BMJ 351: 5876.

31. Qureshi W, Mittal C, Patsias I, Garikapati K, Kuchipudi A, et al. (2014) Restarting anticoagulation and outcomes after major gastrointestinal bleeding in atrial fibrillation. Am J Cardiol 113: 662-668.

32. Witt DM, Delate T, Garcia DA, Clark NP, Hylek EM, et al. (2012) Risk of thromboembolism, recurrent hemorrhage, and death after warfarin therapy interruption for gastrointestinal tract bleeding. Arch Intern Med 172: 1484-1491.

33. Genereux P, Giustino G, Witzenbichler B, Weisz G, Stuckey TD, et al. (2015) Incidence, Predictors, and Impact of Post-Discharge Bleeding After Percutaneous Coronary Intervention. J Am Coll Cardiol 66: 1036-1045. 